\title{
La mise en œuvre d'une stratégie durable \\ - Le cas Biolait, une PME engagée dans le développement durable de la filière laitière biologique -
}

Note de Recherche IREGE $\mathbf{n}^{\circ}$, date 2015

\author{
ANNE MARIE BOCQUET \\ Université de Savoie, France \\ IAE / IREGE \\ 4 ch. de Bellevue \\ BP 80439 - 74944 Annecy-le-Vieux Cedex \\ Anne-Marie.Bocquet@univ-smb.fr
}

Confrontées aux enjeux du développement durable, les entreprises adaptent leurs stratégies. Les stratégies durables, considérées comme le stade le plus avancé d'intégration du développement durable, impliquent une approche proactive, globale et de long terme de l'impact environnemental et social de l'entreprise. Cette définition est envisagée le plus souvent dans le contexte de multinationales globalisées; pourtant, de plus en plus de PME intègrent le développement durable, à un échelon national ou local. Biolait, un groupement de producteurs de lait biologique de l'Ouest de la France, est un cas représentatif d'une PME avec une stratégie durable, orientée autour du développement de la filière laitière biologique française. L'analyse de ce cas montre que cette stratégie engagée ne s'explique pas uniquement par des enjeux de compétitivité. Les enjeux de responsabilité jouent un rôle prépondérant pour expliquer l'ampleur des ambitions économiques, environnementales et sociales de l'entreprise.

Sustainable strategies can be considered as the most advanced stage of integration of sustainable development in the firm; it implies a global, long term and proactive approach of environmental and social impacts. Most of the time, this definition is applied to global firms, in an international context; nevertheless, more and more small and medium firms focus on sustainable development, in a national or a local context. Biolait, a group of organic milk producers in the west of France, is a representative case of a SME implementing a sustainable strategy, based on the development of the French organic milk supply-chain. A qualitative analysis of this case shows that responsibility issues play a more important role than competitive issues, to explain the economic, environmental and social engagement of the firm. 
Confrontées aux enjeux actuels en matière de développement durable, les entreprises sont poussées à intégrer les paramètres environnementaux et sociaux dans leur comportement stratégique. La littérature managériale se préoccupe de ces enjeux et des réponses à apporter, notamment sur le plan écologique, car les entreprises capables d'adapter leur comportement de façon pertinente peuvent en tirer un avantage concurrentiel majeur (Porter, 1991 ; Martinet et Reynaud, 2004 ; Boiral, 2006).

Au-delà des réponses ponctuelles, une véritable intégration du développement durable dans l'entreprise suppose d'évoluer vers l'entreprise durable (Jennings et Zandbergen, 1995 ; Martinet, 2008). Les critères proposés pour définir ces stratégies durables sont centrés sur une approche proactive, globale et de long terme de l'impact environnemental et social de l'activité de l'entreprise (voir par exemple Hart et Milstein, 1995, 1997, 2003). De façon implicite, ces définitions concernent les multinationales globalisées. Or de plus en plus de PME, qui opèrent à un échelon national ou même local, sont concernées par le développement durable et l'intègrent dans leur stratégie (Nicolas, 2004 ; Berger-Douce, 2006). Par ailleurs, il y a peu d'études de cas d'entreprises qui adoptent une démarche de développement durable (Durif et al., 2009). Sur un plan empirique, des analyses de cas concrets se révèlent donc utiles, notamment concernant des entreprises d'envergure nationale ou locale, afin de voir si les critères proposés pour définir les stratégies durables peuvent s’appliquer dans ce contexte.

Le secteur agroalimentaire constitue un terrain d’application idéal de ce type d'études car il est concerné au premier chef par les enjeux de développement durable. A cause d’importants problèmes environnementaux, liés au fonctionnement actuel du système agroalimentaire dans les pays développés, on est loin d’un «développement agroalimentaire durable» (Rastoin et Guersi, 2010). Au premier rang des préoccupations figure l'utilisation excessive d'engrais et de pesticides chimiques, qui pose des problèmes de pollution et 
concernant les risques alimentaires (Moletta, 2006; Bergadaa et Urien, 2006). L’agriculture biologique, qui refuse l'emploi des produits de synthèse, peut être considérée comme une solution pour évoluer vers « une agriculture durable » (Roger, 2002). Le développement de ce type d'agriculture en France représente un enjeu de développement durable car la France est le $2^{\text {ème }}$ consommateur de produits phytosanitaires au monde et exploite à peine 2,5 \% de sa Surface Agricole en agriculture biologique (Agence Bio, 2010).

Dans ce contexte, le groupement de producteurs Biolait, engagé depuis sa création dans le développement de la filière laitière biologique en France, est un cas intéressant pour analyser la mise en œuvre d’une stratégie durable à un échelon local et national. L'entreprise est une PME qui assume un rôle moteur dans la filière, en développant la collecte de lait biologique (principalement dans l'Ouest de la France), et par la représentation des producteurs dans les instances nationales.

Une méthodologie qualitative nous a permis d’analyser la stratégie suivie par Biolait dans la filière laitière biologique. Dans un premier temps, nous présentons le cadre théorique, qui concerne l'intégration du développement durable dans les stratégies des entreprises, puis nous introduisons le cas et développons la méthodologie suivie, avant de présenter et discuter les résultats de l’analyse du cas.

\section{I - L'INTEGRATION DU DEVELOPPEMENT DURABLE DANS LES STRATEGIES DES ENTREPRISES}

Le concept de développement durable, qui cherche à orienter le développement économique vers la prise en considération des enjeux environnementaux et sociaux, concerne les entreprises. Celles-ci intègrent le développement durable car il est source d’enjeux stratégiques auxquels il leur faut apporter une réponse. 


\section{Le concept de développement durable}

Le développement durable a été défini en 1987 dans le rapport Bruntland de la Commission Mondiale Environnement et le Développement : c’est un développement qui répond aux besoins du présent sans compromettre la capacité des générations futures à répondre aux leurs (WCED, 1987). On distingue trois volets dans le développement durable : le progrès économique, la justice sociale et la préservation de l’environnement, mais cette « rhétorique des trois piliers » ne dit rien sur l'articulation entre les trois volets, si ce n'est sous la forme d’un hypothétique équilibre. Le développement durable apparaît donc comme l’un des paradigmes contemporains les plus prometteurs mais aussi les plus flous (Vivien, 2003 ; Boiral et Croteau, 2004).

L’intérêt accordé actuellement au développement durable est principalement lié à la prise de conscience des enjeux environnementaux. De façon globale, un constat s'impose : le développement poursuivi actuellement dans les pays développés n’est pas durable sur le plan écologique ${ }^{1}$ (WWF, 2010). Ces constations alarmantes s’appliquent en particulier au secteur agroalimentaire, qui est concerné au premier chef par les enjeux de développement durable car il tire ses produits de la nature. On est loin d'un «développement agroalimentaire durable »; en cause, des externalités négatives importantes, notamment sur le plan environnemental (Rastoin et Guersi, 2010). Les enjeux écologiques majeurs concernent aussi bien la consommation et les risques alimentaires que la production et ses rejets (Moletta, 2006 ; Bergadaa et Urien, 2006). L’utilisation d'engrais et de pesticides chimiques dans l'agriculture intensive est particulièrement préoccupante, notamment en France, aussi bien au niveau des risques pour la santé que de la pollution des sites agricoles.

\footnotetext{
1 Aucun pays n’atteint un niveau de développement élevé tout en conservant un impact environnemental inférieur au seuil de durabilité écologique ; il faudrait par exemple entre 2 et 3 planètes Terre, si l'ensemble de la planète suivait le mode de développement des pays européens.
} 


\section{Les stratégies des entreprises orientées autour du développement durable}

Ces préoccupations concernent les entreprises, qui, par leur comportement, jouent un rôle clé pour atteindre un développement durable, notamment sur le plan écologique (Shrivastava, 1995). L’intégration du développement durable suppose de définir des critères de durabilité dans l'entreprise, et d'évoluer vers l'entreprise durable (Jennings et Zandbergen, 1995 ; Martinet, 2008 ; Werbach, 2009)². Trois critères sont proposés dans la littérature :

- Un premier critère considère l'entreprise durable comme une entreprise proactive sur le plan écologique, qui met en oeuvre des changements radicaux dans sa stratégie (Jennings et Zandbergen, 1995 ; Hart, 1995, 1997). Cette attitude peut être la source d'un avantage concurrentiel majeur (Porter, 1991 ; Martinet et Reynaud, 2004 ; Boiral, 2006).

- Un deuxième critère envisage la durabilité dans l'entreprise comme la recherche d’un équilibre entre les trois volets du développement durable, en créant de la valeur simultanément sur les trois plans ou en arbitrant entre les trois piliers (Wheeler et al., 2003 ; Hart et Milstein, 2003).

- Un troisième critère considère les stratégies durables comme le stade le plus avancé d’intégration du développement durable (Hart, 1995, 1997 ; Hart et Milstein, 2003). Cela implique la prévention de la pollution (réduction des émissions générées par les activités de production), un management éco-responsable du produit (prise en compte de l'ensemble des impacts environnementaux et sociaux des produits « du berceau à la tombe »), et une approche globale et de long terme de l'impact environnemental et social de l'entreprise (y compris dans les pays du Sud).

De façon implicite, par les exemples choisis ou le champ d’action impliqué, ces définitions sont envisagées dans le contexte de multinationales globalisées. Or de plus en plus de PME

\footnotetext{
${ }^{2}$ Ce qui n’est pas simple car la définition proposée par le rapport Bruntland, large et peu précise, ne facilite pas une définition claire et adaptée aux stratégies des entreprises (Boiral et Croteau, 2004).
} 
sont concernées par le développement durable et l’intègrent dans leur stratégie, à un échelon national, voire local ${ }^{3}$.

\section{Les enjeux de compétitivité et de responsabilité qui expliquent l'intégration du développement durable dans les stratégies}

Plusieurs courants théoriques permettent d'expliquer la prise en compte du développement durable dans l'entreprise, par des enjeux de compétitivité ou de responsabilité.

Les enjeux de compétitivité du développement durable sont relatifs à :

- L’existence de contraintes réglementaires (taxes, réglementation...) et/ou d’opportunités de baisse des coûts (car la pollution constitue un gaspillage) ; l'entreprise a alors intérêt à réduire son impact environnemental pour améliorer sa compétitivité (perspective « win-win ») (Porter, 1991 ; Boiral, 2006).

- Des comportements d’achat qui intègrent la dimension écologique, ce qui génère des opportunités (ou des menaces) marketing (Binninger et Robert, 2008).

- La réponse de l'entreprise aux attentes de ses « parties prenantes » ${ }^{4}$, par exemple les ONG, les médias, les associations de consommateurs ou de protection de l'environnement (Freeman, 1984 ; Mercier, 2006).

- La volonté d'exploiter ou développer des ressources et des compétences environnementales clés détenues par l’entreprise (Persais, 2001; Bowen et Sharma, 2005). La prise en compte du développement durable par les entreprises s’explique également par des enjeux de responsabilité. L’idée selon laquelle l'entreprise est responsable envers la société n’est pas nouvelle ${ }^{5}$, et est largement admise à l’heure actuelle ${ }^{6}$. Concrètement, cette responsabilité s’exerce :

\footnotetext{
${ }^{3}$ Sur l'engagement des PME dans le développement durable, voir par exemple Berger-Douce (2006), ou encore Nicolas (2004) dans le contexte de l'agriculture biologique.

${ }^{4}$ Ce sont les acteurs qui peuvent affecter ou être affectés par la réalisation des objectifs de l'organisation.

${ }^{5}$ On peut en faire remonter l'origine aux travaux de Bowen dans les années 50.
} 
- Envers les parties prenantes de l'entreprise, qui ont des attentes environnementales ou sociales ${ }^{7}$ (Mercier, 2006).

- Envers la nature, ce qui implique de prendre en considération l'environnement naturel en tant que tel (Bazin, 2006).

- Envers le développement durable, ce qui implique une réflexion globale orientée sur le long terme et la prise en considération des générations futures (Lauriol, 2004). En Europe, on assimile responsabilité et contribution de l'entreprise aux trois plans du développement durable (Capron et Quairel-Lanoizelée, 2004 ; Perez, 2005).

\section{LE CONTEXTE DE L’ETUDE ET LA METHODOLOGIE SUIVIE}

Le cas Biolait est pertinent pour notre étude, du fait de l'engagement de cette PME, à rayonnement régional et national, dans le développement de la filière laitière biologique en France ; une étude approfondie du cas a été menée.

\section{Une PME fortement engagée dans le développement de l’agriculture biologique}

Biolait est un groupement de producteurs de lait biologique de l'Ouest de la France, créée en 1994 afin de collecter et commercialiser du lait issu de l'agriculture biologique. C'est une PME, mais l'entreprise s’impose comme le $1^{\mathrm{er}}$ collecteur de lait biologique indépendant sur le territoire français. Le choix de Biolait comme cas représentatif d’une stratégie durable se justifie par l'engagement de l'entreprise dans l'agriculture biologique, avec un projet à long terme de développement de la filière laitière.

La stratégie de Biolait est en effet orientée autour du développement de l'agriculture biologique, un choix de conviction (la structure collecte exclusivement du lait issu de ce mode de production). Or, l’agriculture biologique peut être considérée comme une solution

\footnotetext{
${ }^{6}$ On peut parler de responsabilité sociétale ou globale (Capron et Quairel-Lanoizelée, 2004 ; Bazin, 2006).

${ }^{7} \mathrm{La}$ « théorie des parties prenantes » constitue d'ailleurs la référence théorique centrale des travaux anglosaxons qui portent sur l'éthique organisationnelle et la responsabilité de l'entreprise (Mercier, 2006).
} 
pour répondre aux enjeux de développement durable auxquels est confronté le secteur agroalimentaire et évoluer vers une agriculture durable (Roger, 2002). La filière biologique refuse l'emploi des engrais et pesticides chimiques, et privilégie des moyens naturels pour lutter contre les ravageurs ${ }^{8}$. Les objectifs affichés par les promoteurs de ce mode de production sont à la fois de fournir des produits de qualité, de respecter l'environnement et de favoriser un développement agricole moins intensif (en privilégiant des exploitations de petite taille et autosuffisantes). En France, le label Agriculture Biologique (logo AB) garantit le respect des principes de l'agriculture biologique. La France exploite à peine 2,5\% de sa Surface Agricole selon les critères de l'agriculture biologique ${ }^{9}$ (Agence Bio, 2010). Le développement de ce type d’agriculture en France représente donc un enjeu à la fois environnemental, social, et économique ${ }^{10}$.

Dans ce contexte, Biolait mène un projet ambitieux de développement sur le créneau biologique du secteur laitier. La stratégie de l'entreprise est orientée autour d'un développement durable de la filière laitière biologique française, avec la volonté de favoriser un développement économiquement pérenne et solidaire de cette filière, en assurant une rémunération équitable pour tous les producteurs. Ce « projet de producteurs » est à l'origine de la création de l'entreprise, avec pour objectifs de développer la collecte de lait biologique, de structurer la filière, d'accompagner les producteurs dans leurs projets et de les représenter dans les instances nationales.

\footnotetext{
${ }^{8}$ Ce mode de production interdit également les OGM et limite le recours aux médicaments vétérinaires pour soigner les animaux.

${ }^{9}$ La France, pourtant $1^{\text {ère }}$ puissance agricole européenne, se place ainsi seulement à la $20^{\text {ème }}$ place au niveau européen, voir par exemple Eurostat, L'agriculture biologique dans l'UE-25 - Statistiques en bref, Communautés européennes, 2007; en ligne site internet Eurostat : http://epp.eurostat.ec.europa.eu/cache/ITY_OFFPUB/KS-SF-07-069/FR/KS-SF-07-069-FR.PDF

${ }^{10}$ Dans la filière laitière, le développement de la production biologique peut être une des pistes pour faire face à la crise qui affecte le secteur laitier, depuis l'abandon des régulations publiques par les quotas laitiers et les prix indicatifs.
} 


\section{Présentation du cas Biolait}

Avec un CA de plus de 20 Millions d' $€^{11}$, Biolait est le $1^{\text {er }}$ collecteur indépendant de lait biologique en France. Créée à l'initiative de six producteurs de l’Ouest, Biolait collecte maintenant presque partout sur le territoire français ${ }^{12}$.

Biolait est un groupement qui fonctionne selon le principe « un homme, une voix ». Dès le départ, Biolait affirme des ambitions environnementales et sociales, et son engagement pour le développement de la filière laitière biologique. C'est un projet à l'origine de la création de la structure, puisque l'organisation de la collecte est alors perçue comme le principal frein au développement de la filière. Biolait se veut un «projet de producteurs » qui s’engagent pour « un développement solidaire de la filière lait biologique »; c’est une « démarche à vocation économique, humaine et environnementale », traduite dans le slogan « La Bio partout et pour tous ${ }^{13}$. Ces ambitions se traduisent dans les objectifs de départ :

- Encourager le développement de l'agriculture biologique partout et pour tous, en collectant le maximum de lait biologique sur toutes les zones avec le même prix du lait pour tous (c’est la «mutualisation » du prix du lait)

- Participer à l'organisation de la filière en mettant en adéquation offre et demande

- Représenter les producteurs de lait biologique

- Développer l’offre de produits laitiers biologiques en livrant à la carte les transformateurs

- Garantir une traçabilité totale.

\footnotetext{
${ }^{11}$ Ce qui représente 300 exploitations agricoles sur 53 départements et 40 salariés en 2009.

${ }^{12}$ La structure a connu un fort développement les premières années après sa création, a traversé une crise entre 2002 et 2005 (liée aux difficultés dans la filière laitière biologique), et connaît à nouveau une phase de croissance depuis 2006 (en 2009, la demande de lait bio en France est en forte augmentation et tire la production, alors que les prix du lait conventionnel chutent).

${ }^{13}$ Voir site internet ; «Biolait : $1^{\mathrm{er}}$ collecteur de lait biologique » (2004) ; « Biolait - Une démarche à vocation économique, humaine et environnementale » (2010).
} 


\section{Encadré méthodologique}

- Méthodologie : qualitative (étude de cas), à visée descriptive et explicative.

- Critère de sélection du cas : potentiel de découverte lié à la richesse du cas.

- Sources de données : diversifiées (objectif de triangulation des données) :

* Entretiens : dans et à l'extérieur de l'entreprise :

Entretiens semi-directifs (auprès du Directeur, de la Responsable Communication et de la Responsable Commerciale), enregistrés (d'1h30 en moyenne); un entretien auprès d'un chargé de mission CRIEL ${ }^{14}$ (pour comprendre les enjeux de la filière laitière et de l'AB).

* Sources documentaires ${ }^{15}$ : internes et externes :

Analyse de documents internes et externes publiés par Biolait, d’éléments émanant de la profession (dans des revues professionnelles, des sites internet) qui citent l'expérience Biolait (celle-ci bénéficiant d'une certaine notoriété). Par ailleurs, un ouvrage consacré à l'agriculture biologique et la consom'action évoque l'entreprise (Rimsky-Korsakoff, 2003).

\section{- Recueil de données :}

Contenu et objectifs : les données recueillies portent sur la stratégie menée par Biolait, la perception des évolutions au sein de la filière laitière biologique, les enjeux de compétitivité de l'engagement de l'entreprise et sa responsabilité (envers la nature, les parties prenantes et le développement durable). Les entretiens permettent de recueillir des verbatims. Les données externes apportent un regard extérieur sur l’expérience de Biolait. Période de recueil : les données, obtenues au cours de deux périodes successives d'étude, concernent une période assez longue (entre 2004 et 2010), ce qui facilite le recul sur la stratégie menée et la prise en compte des évolutions de la filière laitière biologique.

\section{- Cadre d'analyse et élaboration des résultats :}

Codification des données et analyse de contenu en respectant certains principes d'analyse (par exemple un effort de contextualisation des données). L’étude du cas a été menée en deux étapes : identification de la stratégie suivie (à partir des critères de durabilité), puis compréhension, à partir des enjeux de compétitivité et de responsabilité.

\footnotetext{
${ }^{14}$ Le CRIEL est le Centre Régional Interprofessionnel de l’Economie Laitière.

${ }^{15}$ Biolait : $1^{\text {er }}$ collecteur de lait biologique, avril 2004

Biolait - Une démarche à vocation économique, humaine et environnementale -, 2010 en ligne site internet de Biolait : http://www.biolait.net/Fr/fiche\%20de\%20presentation\%20pdf.html

Rimsky-Korsakoff, Au delà du bio : la consom'action, Editions Yves Michel, Barret-sur-Méouge, 2003.
} 


\section{LA STRATEGIE DURABLE SUIVIE PAR BIOLAIT - UN DEVELOPPEMENT}

PERENNE ET SOLIDAIRE DE LA FILIERE LAITIERE BIOLOGIQUE FRANÇAISE -

La stratégie menée par Biolait peut être considérée comme durable ; elle répond aux enjeux de compétitivité et de responsabilité identifiés par l’entreprise.

\section{L’analyse de la stratégie menée : une stratégie de développement durable}

Tous les critères qui permettent de définir une stratégie durable sont réunis, ici à un échelon régional / national : la proactivité écologique, l’intégration du volet social, la prévention de la pollution et le management éco-responsable du produit, une approche globale et de long terme de l'impact environnemental et social de l'entreprise (sur son territoire d'action).

\section{Proactivité écologique}

Biolait assume un rôle moteur dans le développement de la filière laitière biologique. Cette attitude proactive a été à l’origine de la création de la structure en 1994, et est restée de mise depuis. Même dans les périodes de baisse du prix du lait, Biolait a cherché à développer le réseau d'adhérents afin d’intensifier l’activité de collecte du lait biologique.

\begin{tabular}{|l|}
\hline "Pourquoi avoir créé Biolait ? Nous avons créé cette structure, fin 1994, afin de \\
promouvoir le développement de la filière laitière biologique. " "Nous nous \\
sommes engagés dans cette entreprise risquée et difficile car nous avons été les \\
premiers à croire en l'avenir et au bien fondé de ce mode de production. " \\
(Présidente, dans Biolait : $1^{\text {er }}$ collecteur de lait biologique, 2004) \\
\hline "Les adhérents de Biolait ont développé le groupement sur une large partie du \\
territoire pour devenir la plus importante structure économique de producteurs \\
collectant exclusivement du lait biologique. "(Biolait, une démarche à vocation \\
économique, humaine et environnementale, 2010)
\end{tabular}

Cette attitude proactive vise à jouer un rôle d'entraînement envers les autres acteurs de la

filière : producteurs de lait, transformateurs, et également les consommateurs.

«Au niveau de Biolait (...) on cherche à développer la bio, structurer la filière. Le développement de la bio, c'est aussi bien développer la production en permettant au maximum d'en vivre, développer la transformation et la consommation, pour fournir le maximum de consommateurs. » (Responsable Communication)

"On a un objectif ambitieux : collecter plus de 70 Millions de litres de lait à 5 ans ! Soit 6 Millions de plus par an, en raccrochant des producteurs qui souhaitent se convertir, et des producteurs bio qui veulent venir chez nous. » (Directeur) 


\section{Intégration du volet social et recherche d'équilibre entre les trois volets}

Le projet affiché par Biolait est à double vocation environnementale et sociale : existence d’un lieu d'échange sur les pratiques agricoles entre les producteurs, soutien aux projets des producteurs, solidarité (avec une équité du prix du lait quelle que soit la zone de production, ce qui permet à tous de valoriser leur production biologique). La solidarité sociale entre les producteurs (même prix du lait pour tous) démontre une volonté d’équilibre entre le volet social et le volet économique.

\begin{tabular}{l}
\hline "Notre projet: (...) solidarité au sein de la filière laitière biologique avec des \\
impératifs d'équité (...) : entre les producteurs de Biolait, quels que soient le \\
volume produit ou la situation géographique du producteur, le prix payé est le \\
même pour tous les adhérents. » (site internet) \\
\hline "Biolait va partout, même dans les zones les plus isolées, cela répond à cette \\
notion de solidarité. »; "Le fait d'être en bio fait partie d'une démarche durable, \\
de préservation des ressources... le fait d'être à Biolait c'est plus le côté social (le \\
même prix du lait pour tous), des choix classiques pourraient être plus \\
rémunérateurs. » (Responsable Communication) \\
\hline "La part éthique est très importante; la notion de solidarité a été prouvée; dans \\
les périodes de vaches maigres, les producteurs ont été mutualisés. Le lait a été \\
collecté par des collecteurs conventionnels, mais Biolait a assuré le paiement \\
d'une surprime bio.» (Directeur)
\end{tabular}

\section{Prévention de la pollution et management éco-responsable du produit}

L’agriculture biologique, le mode de production exclusif au sein de Biolait, est considérée comme la meilleure garantie pour minimiser les rejets au niveau des exploitations, mais aussi pour assurer l’absence de résidus phytosanitaires dans les produits.

\begin{tabular}{|l|}
\hline En terme de préservation de l'environnement, l'agriculture biologique c'est le \\
meilleur choix ; pour démontrer le contraire, il faut s'accrocher! Il n'y a qu'à voir \\
concernant l'état sanitaire de l'eau, certaines collectivités sont prêtes à mettre la \\
main au portefeuille pour développer l'agriculture biologique, aider les \\
producteurs à se convertir!» (Directeur) \\
\hline «La bio a sa place et devrait en avoir plus, cela passe par une sensibilisation \\
beaucoup plus forte du consommateur. En France, on est assez frileux sur les \\
informations (les impacts des produits phytosanitaires...); le peu de choses qui \\
sortent ont un effet, cela touche quand même les gens. En Angleterre, une \\
organisation du type de Biolait a une communication beaucoup plus agressive : les \\
produits phyto, c'est tant de résidus que vous absorbez...» (Responsable \\
Communication)
\end{tabular}




\section{Approche globale et à long terme de l'impact environnemental et social de}

\section{l'entreprise sur son territoire d'action}

La stratégie de l'entreprise implique par ailleurs une approche globale et de long terme de son rôle, à un échelon régional et national. L’entreprise assume ainsi une véritable démarche de développement durable de la filière laitière biologique en France (créée par des producteurs de l’Ouest, Biolait est aujourd’hui présent sur tout le territoire français).

«Un projet de producteurs, un développement national : en 1994, alors que
l'agriculture biologique est encore, en France, une niche sans intérêt économique
(...), une poignée d'éleveurs laitiers biologiques de l'Ouest fait le pari de lever le
principal frein au développement de la filière laitière biologique : l'organisation
de la collecte. Fortement convaincus de l'avenir et du bien fondé de l'agriculture
biologique, les adhérents de Biolait ont, petit à petit, développé le groupement sur
une large partie du territoire (...)» (Biolait, une démarche à vocation économique,
humaine et environnementale, 2010)

Cette approche se concrétise par l'influence de Biolait sur le marché du lait biologique, son rôle en matière de développement local, d’aménagement du territoire, et de représentation au sein des structures régionales et nationales.

\begin{tabular}{l}
\hline «A niveau des producteurs, il y a une reconnaissance du rôle de Biolait, de son \\
importance depuis sa création, son rôle joué dans le développement du marché \\
on a pesé sur le marché et le prix de Biolait s'impose comme référence du prix du \\
lait. » (Responsable Communication) \\
\hline "Biolait, acteur du développement local et de l'aménagement du territoire. " \\
(Biolait : $1^{\text {er }}$ collecteur de lait biologique, 2004) \\
\hline "Notre projet : (...) reconnaissance et développement de l'agriculture biologique. \\
Par leur présence dans diverses instances régionales et nationales (FNAB ${ }^{16}$, \\
Biocoop...), les adhérents de Biolait ont la volonté de favoriser un développement \\
durable de leur production. » (site internet)
\end{tabular}

\section{Les enjeux de compétitivité et de responsabilité de la stratégie suivie}

Cette stratégie durable répond aux enjeux identifiés par Biolait : enjeux de compétitivité (qui apparaissent insuffisants pour expliquer la stratégie suivie) et de responsabilité.

\section{- $\quad$ Contraintes réglementaires et opportunités de réduction des coûts}

L’agriculture biologique apporte une réponse aux préoccupations écologiques des pouvoirs publics, mais le label est beaucoup plus contraignant que les obligations réglementaires

\footnotetext{
${ }^{16}$ Fédération Nationale de l’Agriculture Biologique.
} 
actuelles. Par ailleurs, le respect des règles de l'agriculture biologique ne permet pas de réduire les coûts ; on observe au contraire des surcoûts (par rapport à l'agriculture conventionnelle et à l'agriculture raisonnée), pas toujours faciles à répercuter sur le consommateur ${ }^{17}$.

\section{- $\quad$ Opportunités marketing}

La demande de produits laitiers biologiques crée des opportunités, mais elle rencontre de nombreux freins. Le marché est instable et Biolait, qui reste un petit acteur face aux groupes industriels laitiers, est fortement impacté par ces évolutions.

"Les consommateurs sont plus en alerte sur l'environnement, la sensibilité est plus forte aujourd'hui, mais ne se traduit pas forcément par un acte d'achat bio ! Il y a des freins: les habitudes de consommation, le prix, la disponibilité... (Responsable Communication)

"Il y a eu une crise en 2002-2005, une période difficile avec une surproduction de lait bio, peu de demande, ce qui a créé un déséquilibre de marché ; ce lait bio a atterri dans la filière conventionnelle, avec une piètre rémunération. Aujourd'hui, la donne a changé, les cours laitiers ont remonté, le cours du lait bio se maintient, la demande de lait bio est au rendez-vous.» (Directeur)

\section{- $\quad$ Attentes des parties prenantes}

L’intérêt des parties prenantes (medias, grande distribution...) pour l’agriculture biologique existe, mais évolue au gré des tendances.

"En 1997-98, il n'y avait pas une semaine sans un article sur les produits bio ; beaucoup de transformateurs ont alors décidé de partir sur le bio. C'est moins le cas à l'heure actuelle. Aujourd'hui, la grande distribution n'est plus moteur, elle est assez attentiste... » (Responsable Commerciale)

\section{- $\quad$ Ressources et compétences environnementales}

Le savoir-faire des adhérents de Biolait (en terme de pratiques agricoles et de gestion) est réel, mais pour Biolait, l'engagement dans la filière biologique ne dépend pas uniquement de ces compétences ; il reflète davantage un choix de conviction.

"Il y a le marché, des compétences de gestion, qui sont venus après, mais au départ, l'agriculture biologique, c'était vraiment un choix politique. " (Responsable Communication)

\footnotetext{
${ }^{17}$ L’AB permet des économies au niveau des intrants (engrais et pesticides chimiques), mais entraine des surcoûts en main d'œuvre, liés par exemple au désherbage et à l'entretien des cultures.
} 
La stratégie de développement durable menée par Biolait ne se justifie donc pas uniquement par la réponse à des enjeux de compétitivité ; les enjeux en matière de responsabilité de l'entreprise (envers la nature, les parties prenantes et le développement durable) jouent un rôle plus important.

- La responsabilité envers la nature : le choix exclusif de l'agriculture biologique

Le souci de préserver l'environnement naturel explique le choix de l'agriculture biologique, un choix politique affiché comme une condition d'appartenance à la structure. Biolait rejoint ici le point de vue des acteurs de la filière laitière, pour qui l'agriculture biologique correspond avant tout à une volonté de protection de l'environnement.

"(L'agriculture biologique) c'est un choix au départ, de politique; on ne travaille que le lait bio, on a un objectif de développement du bio inscrit dans les statuts. » «Ne pas utiliser de pesticides de synthèse, c'est quelque part un choix ultime pour la protection de l'environnement.» (Responsable Communication)

«Le bio n'est pas une garantie de santé, mais une garantie de protection de la nature avant tout! » (Chargé de mission CRIEL ${ }^{18}$ )

Cette responsabilité assumée envers la nature prend le pas sur les préoccupations économiques pour expliquer le choix du mode de production biologique et l'engagement au sein de la filière. Le choix exclusif de l’agriculture biologique n’a d’ailleurs jamais été remis en question depuis la création de l'entreprise, quelle que soit l'évolution du marché.

\begin{tabular}{|l|}
\hline "Nous avons créé cette structure, fin 1994, afin de promouvoir le développement \\
de la production laitière biologique. L'AB était encore à cette époque perçue \\
comme une niche de marché sans réel intérêt économique.» (Présidente de \\
Biolait, dans Biolait : $1^{e r}$ collecteur de lait biologique, 2004). \\
\hline "C'est ainsi qu'est né Biolait. De petits éleveurs isolés se sont regroupés et ont \\
acheté un camion-citerne pour collecter le lait. En effet, faire tourner une citerne \\
sur $200 \mathbf{k m}$ de routes de montagnes pour collecter une poignée de marginaux sur \\
leur nid d'aigles n'était pas rentable pour un groupe laitier! Certains ont ainsi \\
attendu 10 ans avant de pouvoir vendre et valoriser leur lait en bio. " (Rimsky- \\
Korsakoff, 2003, p. 142) \\
\hline "(Pour les producteurs) le fait d'être en bio fait partie d'une démarche durable, \\
de préservation des ressources. " "Si ils regardaient uniquement le marché, ils \\
n'auraient pas forcément envie d'aller vers le bio. » (Responsable Communication) \\
\hline
\end{tabular}

\footnotetext{
${ }^{18}$ Le CRIEL est le Centre Régional Interprofessionnel de l’Economie Laitière.
} 
- La responsabilité envers les parties prenantes : un engagement social envers les producteurs

Biolait est un groupement de producteurs géré sur la base « un homme, une voix ». La structure affiche un principe d'équité et de transparence, et assume une responsabilité sociale envers l'ensemble des producteurs, avec la recherche de relations plus solidaires.

"Pour les producteurs: un principe d'équité (le même montant de parts sociales,
un prix payé identique), une structure gérée par les producteurs avec un
fonctionnement transparent et démocratique (toutes les décisions d'ordre politique
ou stratégique sont prises par les producteurs) (Biolait, une démarche à vocation
économique, humaine et environnementale, 2010)
"Le projet de Biolait part d'une forte conscience environnementale, et instaurer
des relations différentes entre les différents acteurs de la filière, des relations
commerciales plus humaines, la solidarité entre les adhérents (le même prix pour
tous, aller chercher le lait sur tout le territoire malgré les coûts), une démarche
globale dans le bio. » (Responsable Communication)

La responsabilité de la structure collective envers les producteurs se concrétise par un accompagnement économique (soutien aux projets collectifs de développement dans la filière biologique) et social (mutualisation du prix du lait entre les producteurs). Une structure, la Voix Biolactée, a été créée pour accompagner les projets de développement.

«La Voix Biolacté, le lieu privilégié d'échange entre les adhérents, propose les
actions suivantes: communication bio vers le monde agricole et ses filières,
accompagnement des initiatives locales (... soutenir, accompagner et coordonner
les projets collectifs initiés par ses membres.)» (Biolait, une démarche à vocation
économique, humaine et environnementale, 2010)

La mutualisation du prix du lait est une démarche de solidarité particulièrement significative de l'engagement social de l'entreprise ; c'est l'une des forces de Biolait. Ce mécanisme permet à tous les producteurs de toucher le même prix pour leur lait, quelque soit la zone de collecte et la situation du marché ${ }^{19}$. Cette solidarité est la garantie du maintien du réseau de producteurs, notamment dans les périodes de baisse du prix du lait.

«La part éthique est très importante (...) dans les périodes de vaches maigres, les producteurs ont été mutualisés. Le lait a été collecté par des collecteurs conventionnels, mais Biolait a assuré le paiement d'une surprime bio. (...) Ca a été un succès ; la mutualisation du prix du lait, dans les périodes de vaches maigres, permet au réseau de se maintenir et de se développer.» (Directeur)

\footnotetext{
19 y compris ceux dont le lait ne peut pas être valorisé dans la filière biologique (pour des raisons
} géographiques ou liées à la baisse de la demande). 


\section{- La responsabilité dans le développement durable de la filière laitière biologique}

Dès sa création, l’entreprise affiche des convictions fortes, une responsabilité au sein de la société. Celle-ci se traduit par des ambitions économiques, sociales et environnementales, les trois volets du développement durable perçus comme complémentaires.

\begin{tabular}{l} 
«Les producteurs, c'est d'abord un choix philosophique chez Biolait, de vie, de \\
société, un choix éthique. Il faut faire la conjonction avec l'économique; les deux \\
ne sont pas antinomiques. » (Directeur) \\
\hline "(Biolait est) un projet économique qui s'appuie aussi sur des convictions fortes, \\
politiques, idéologiques. "; "C'est une volonté d'apporter une pierre à des \\
relations différentes entre les gens, un côté plus social et humain dans la société. " \\
(Responsable Communication)
\end{tabular}

Cet engagement est un point clé pour comprendre le projet de l’entreprise, orienté autour d’un développement sur le long terme de la filière laitière biologique, lié à des convictions d’ordre politique qui s’inscrivent dans une vision de la société.

«On fait des choix depuis 10 ans (...). Le bio participe à changer les choses, correspond à notre vision de la société.»; "Le fait de développer la collecte partout correspond à cette volonté de développement durable car ça encourage au développement de la bio. » (Responsable Communication)

\section{Synthèse et discussion des résultats}

La stratégie suivie par Biolait est une stratégie durable, selon tous les critères définis :

- La proactivité écologique, avec le rôle moteur de Biolait dans le développement de la filière laitière biologique.

- La recherche d’équilibre entre les trois volets du développement durable, particulièrement significative ici, et une démarche de création de valeur simultanée sur les trois plans : économique, social et environnemental (au sens de Wheeler et al., 2003).

- L'approche globale et à long terme de l'impact environnemental et social de l'entreprise, qui assume un rôle d'acteur du développement de la filière laitière biologique française dans la durée.

Biolait est donc un exemple représentatif de la mise en œuvre d’une stratégie durable dans une PME d'envergure régionale / nationale, et démontre que ce type de stratégie n’est pas 
réservé aux multinationales globalisées. Le cas s’insère dans une série de travaux consacrés à l’intégration du développement durable dans les PME (voir Nicolas, 2004 ${ }^{20}$; Berger-Douce, 2006), et permet d’élargir le champ des définitions des stratégies durables. Pour justifier le choix de ce type de stratégie par l’entreprise, les enjeux proposés dans la littérature ont servi de grille de lecture :

Des enjeux de compétitivité ont été identifiés, mais ceux-ci restent insuffisants pour expliquer l’engagement de Biolait dans le développement durable :

- L'agriculture biologique ne correspond pas à une obligation réglementaire et ne permet pas de réduire les coûts.

- Les opportunités marketing liées à la demande de produits laitiers biologiques subissent d'importantes fluctuations.

- L’intérêt des parties prenantes pour le bio évolue au gré des tendances.

- L’exploitation de compétences environnementales ne justifie pas l'engagement de Biolait, davantage un choix de conviction.

Ce sont les enjeux liés à la responsabilité de l'entreprise qui jouent un rôle majeur pour comprendre l’engagement stratégique de Biolait dans le développement durable :

- La responsabilité envers la nature, avec le choix exclusif de l'agriculture biologique.

- La responsabilité envers les parties prenantes, par la mutualisation du prix du lait pour les producteurs (la garantie du même prix payé à tous).

- La responsabilité dans le développement durable, avec dès le départ un projet de développement de la filière biologique laitière sur le long terme.

Le cas Biolait permet ainsi de mettre en évidence le rôle des convictions, dans l'entreprise, pour expliquer le choix d'une stratégie durable et les orientations choisies dans le cadre de cette stratégie. Le développement de la filière laitière biologique est un «projet de

\footnotetext{
${ }^{20}$ Nicolas (2004) envisage la démarche agriculture biologique dans les PME comme une traduction des principes du développement durable.
} 
producteurs », un choix de conviction qui n’a pas été remis en question malgré les difficultés économiques au sein de la filière. Biolait fait d’ailleurs partie des défenseurs et promoteurs de l'agriculture biologique ${ }^{21}$, citée en exemple par les acteurs du monde agricole et les défenseurs de l'agriculture biologique (Rimsky-Korsakoff, 2003)22.

Il faut par ailleurs souligner la pérennité économique de la stratégie suivie par Biolait. Deux éléments jouent un rôle clé à ce niveau :

- L'approche globale et à long terme adoptée, qui vise à structurer et développer la filière biologique laitière, ce qui permet de peser sur le prix du lait et l'évolution du marché.

- La complémentarité entre projet économique, environnemental et social, avec la solidarité sociale entre les producteurs par la mutualisation du prix du lait, un mécanisme en rupture avec une logique exclusivement économique et de court terme. Cette solidarité permet à un maximum de producteurs de pérenniser leur choix de l'agriculture biologique, et assure le maintien et le développement du réseau de collecte.

Ces choix ont permis à la structure de faire face aux fluctuations du marché et de profiter de la phase de croissance actuelle. La stratégie durable menée par l'entreprise est donc particulièrement représentative de la pérennité d’un modèle économique dans lequel les variables sociales et environnementales jouent un rôle important.

\section{CONCLUSION}

A partir du cas Biolait, il a été possible d’appliquer les critères de durabilité proposés dans la littérature (voir Hart et Milstein, 1995, 2003 ; Wheeler et al., 2003 ; Boiral, 2006), à un cas de PME d'envergure régionale et nationale. Cette étude, menée dans le contexte de la filière laitière biologique française, se révèle particulièrement pertinente pour envisager une réflexion sur les stratégies durables, car le secteur agroalimentaire est à la recherche d'un

\footnotetext{
21 L'agriculture raisonnée par exemple, considérée comme trop proche du simple respect de la réglementation, n’a jamais été envisagée comme une alternative pertinente.

${ }^{22}$ Revue Nature et Progrès : http://www.natureet progres ; Revue L'avenir Agricole : http://aveniragricole.net
} 
développement agroalimentaire durable (Rastoin et Guersi, 2010), ce qui passe, en France, par la promotion de l'agriculture biologique.

Le cas Biolait est un cas concret intéressant, sur un plan managérial, d’une stratégie durable orientée vers le développement pérenne et solidaire de la filière laitière biologique française. Une méthodologie qualitative nous a permis de décrire et de comprendre la stratégie suivie, à partir des critères de durabilité et des enjeux de ce type de stratégie.

Plusieurs conclusions principales peuvent être tirées. Tout d’abord, les stratégies durables ne sont pas réservées aux multinationales globalisées, puisque Biolait, une PME, met en œuvre ce type de stratégie à l'échelon régional et national. Le cas illustre aussi une forte complémentarité entre projet social et projet environnemental, avec la mutualisation du prix du lait, un mécanisme de solidarité entre les producteurs en rupture avec une logique purement économique et de court terme. Par ailleurs, le cas Biolait montre que les enjeux de compétitivité sont insuffisants pour comprendre le choix d’une stratégie durable ; ce sont les enjeux relatifs à la responsabilité de l'entreprise, et ses convictions, qui jouent un rôle prépondérant. La stratégie suivie par Biolait est néanmoins tout à fait pérenne sur le plan économique ; la complémentarité entre les variables sociales, économiques et environnementales peut être considérée comme une force pour cette PME.

Il ne semble toutefois pas possible de généraliser les résultats de cette recherche, menée avec une méthodologie qualitative. Il faut noter en particulier le caractère particulièrement exemplaire du cas Biolait. Par ailleurs, l'étude menée comporte des limites, et plusieurs types de prolongements sont à envisager. Il serait par exemple pertinent de réaliser une analyse plus poussée des difficultés rencontrées. D’autre part, le recours à des entretiens complémentaires, auprès des producteurs et partenaires de Biolait (transformateurs, distributeurs), des acteurs du monde agricole et de la filière biologique, permettrait d’approfondir l'étude menée et d’apporter un regard plus critique sur la stratégie suivie. 


\section{BIBLIOGRAPHIE}

Agence Bio, Les chiffres de la Bio sont au vert, Dossier de Presse, mai 2010

Bazin D., L'éthique économique, Armand Colin, Paris, 188 p, 2006

Bergadaa M., Urien B., «Le risque alimentaire perçu comme risque vital de consommation. Emergences, adaptation et gestion », Revue Française de Gestion, Vol. 3, p. 127-144, 2006

Berger-Douce S., "Les enjeux stratégiques de l'engagement environnemental des PME", Gestion 2000, Vol. 23, n¹, janvier-février, p. 171-188, 2006

Binninger A.-S., Robert I., "Consommation et développement durable : vers une segmentation des sensibilités et des comportements ", Revue des Sciences de Gestion, janv-fév, n²22, p. 51-59, 2008

Boiral O., "Global warming: should companies adopt a proactive strategy ? », Long Range Planning , Vol.39, n 3, juin, p. 315-330, 2006

Boiral O., Croteau G., « Du développement durable à l'entreprise durable, ou l'effet Tour de Babel », in Guay L. et al. (dir.), Les enjeux et les défis du développement durable - Connaître, décider, agir, Les presses de l’Université Laval, Québec, p. 259-281, 2004

Bowen F., Sharma S., "Resourcing environmental strategy ; behavioral and resource-based perspectives”, Academy of Management Proceedings, Best Conference Paper, p. A1-A6, 2005

Capron M., Quairel-Lanoizelée F., Mythes et réalités de l'entreprise responsable - Acteurs, Enjeux, Stratégies - , La Découverte, Coll. « Entreprise \& Société », Paris, 252 p., 2004

Durif F., Brosseau A., Turcotte C., Wolff L., «L'opérationnalisation des principes du développement durable. Le cas Mountain Equipment Co-op », Revue Française de Gestion, 2009/1, n 190, p. 57-75, 2009

Freeman R.E., Strategic Management : A Stakeholder Approach, Pitman, Boston, 276 p., 1984

Hart S. L., “A natural-resource-based view of the firm”, Academy of Management Review, Vol. 20, n 4, p. 986-1014, 1995

Hart S. L., "Beyond Greening : Strategies for a Sustainable World”, Harvard Business Review, Vol. 75, n 1, January-February, p. 66-76, 1997

Hart S. L., Milstein M., “Creating sustainable value”, Academy of Management Executive, Vol. 17, $\mathrm{n}^{\circ}$ 2, p. 56-67, 2003

Jennings P. D., Zandbergen P. A., "Ecologically sustainable organizations : an institutional approach”, Academy of Management Review, Vol. 20, n 4, p.1015-1052, 1995

Lauriol J., "Le développement durable à la recherche d'un corps de doctrine », Revue française de gestion, Dossier Développement Durable, Vol. 30, n 152, septembre-octobre, p. 137-150, 2004

Martinet A.C., «Gouvernance et management stratégique - une nouvelle science morale et politique », Revue Française de Gestion, n 183, p. 95-110, 2008

Martinet A.C., Reynaud E., Stratégies d'entreprise et écologie, Economica Gestion, Paris, 165 p., 2004

Mercier S., «La théorie des parties prenantes : une synthèse de la littérature », in BonnafousBoucher M., Pesqueux Y., Décider avec les parties prenantes - Approches d'une nouvelle théorie de la société civile, La Découverte, Coll. « Recherches », p. 157-172, 2006

Moletta R. (coord.), Gestion des problèmes environnementaux dans les industries agroalimentaires,

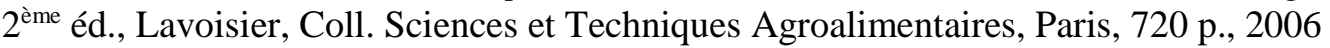

Nicolas E., “Apprentissage organisationnel et développement durable. La norme AB en PME », Revue Française de Gestion, 2004/2, n 149, p. 153-172, 2004

Perez R., «Quelques réflexions sur le management responsable, le développement durable et la responsabilité sociale de l'entreprise ", La Revue des Sciences de Gestion, Direction et Gestion, n 211-212, janvier-avril, p. 29-46, 2005 
Persais E., "Le caractère stratégique des compétences écologiques ", Revue Sciences de Gestion, n 31, hiver, p. 35-56, 2001

Porter M.E., “America’s green strategy”, Scientific American, Vol. 264, nº4, April, p. 96, 1991

Rastoin J.-L., Guersi G., Le système alimentaire mondial - Concepts, méthodes, analyses et dynamiques, Editions Quae, Coll. Synthèses, Versailles, 576 p., 2010

Roger C., "Agriculture raisonnée, multifonctionnelle, biologique,...; quelles voies vers une « agriculture durable », INRA, mensuel, $\mathrm{n}^{\circ} 113$, juin, 2002

Shrivastava P., "The role of corporations in achieving ecological sustainability", Academy of Management Review, Vol. 20, n 4, October, p. 986-1014, 1995

Vivien F.-D., « Jalons pour une histoire de la notion de développement durable », Mondes en Développement, Vol. 31, n 121, 2003

Werbach A., "When sustainability means more than green”, McKinsey Quarterly, n 4, p. 74-79, 2009

Wheeler D., Colbert B., Freeman R.E., "Focusing on Value : Reconciling Corporate Social Responsibility, Sustainability and a Stakeholder Approach in a Network World", Journal of general Management, Vol. 28, n 3, Spring, p. 1-28, 2003

WCED (World Commission Environment and Development) (1987), Our common future, United Nations General Assembly, august 4, 1987

WWF, Global Footprint, ZSL, Planète vivante - Biodiversité, biocapacité, développement -, 2010 